\title{
Arbor
}

\section{El papel de la evolución en la teoría de Daniel Dennett}

\author{
Juan José Colomina Almiñana
}

Arbor CLXXIX, 705 (Septiembre 2004), 247-263 pp.

"Cuesta muchísimo creer que la cola de un pavo real fue formada de esta manera, pero, una vez creído esto, yo creo en el mismo principio, algo modificado, aplicado al hombren, Carta de Darwin a Desmond y Moore (citado en Dennett, 1995: 52).

Tal vez la idea más controvertida de la filosofía de Daniel C. Dennett sea ésta: los seres humanos no son más que mecanismos dotados de intencionalidad derivada configurada por la evolución de las especies. Esto ha sido considerado por algunos como una afrenta porque relega al humano al estatus de simple artefacto: las acciones del ser humano no responden a sus propios intereses sino que se ven programados por «agentes internos especializados para recibir la información disponible en la periferia del cuerpo» ${ }^{1}$ que constituyen. Es decir, el ser humano no es más que un compuesto de pequeños sistemas intencionales organizados según los propósitos de la evolución, propósitos que por otra parte son ciegos. Sus críticos apuestan por la denuncia de esta intencionalidad como falsa: un ser humano dispone de intencionalidad real o intrínseca, de lo contrario no pasaría de ser un mero zombie sin voluntad ni decisión. Esta crítica apuesta también por denunciar la anti-intuitividad de dicha tesis: según el sentido común, los humanos nos sabemos como libres y responsables de nuestros actos, realizamos nuestros movimientos según nuestras propias intenciones.

\section{El mito de la intencionalidad intrínseca}

Darwin revolucionó al mundo al introducir la teoría de la evolución. Dicha teoría afirmaba que todos los seres son producto del proceso de se- 
lección natural iniciado no por una Mente Universal ni por una Inteligencia Divina sino por la acumulación aleatoria y arbitraria de modificaciones ambientales indicadas por algoritmos que permitieron la configuración de un cierto tipo de orden, una rutina, capaz de crear, desarrollar y evolucionar cierto tipo de organismos que consiguieron ele varse hasta la posibilidad de controlar su propio desarrollo.

Este proceso de evolución, en principio arbitrario, acabó por conformar unos organismos con una cierta distribución fenotípica capaz de albergar mentes primitivas (proto-mentes), mecanismos capaces de hacerse cargo de sus propias modificaciones. Esto permitiría explicar de modo sencillo el paso del diseño natural al (re-)diseño inteligente controlado por el mismo individuo. Pero, ¿cuál es el problema?

La explicación naturalista (científica) del nacimiento de la mente humana desmitifica el mundo humano, porque esta tesis desmiente la idea de la folk psychology (psicología popular) que afirma que el ser humano es libre porque es capaz de causar sus propias acciones. En realidad el ser humano no es libre de actuar libremente de este modo porque no elige sus propias acciones: sólo responde a la configuración que realiza por él su propia historia cognitiva, lo que restringe su ámbito de acción.

El desarrollo se produce de modo ciego pero no de modo desordenado. Los organismos primigenios son modificados aleatoriamente para probar su resistencia al entorno en que habitan: aquellos diseños que funcionen se potenciarán, los que no funcionen tenderán a desaparecer. En palabras de Henry W. Beecher, «el diseño al por mayor es de mayor grandeza que el diseño al por menor» (citado en Dennett, 1995: 100). Pero, si el supuesto orden no viene establecido por una intencionalidad superior, ¿cómo pudieron evolucionar los organismos mejor diseñados? Esto es, si negamos la existencia de lo que Locke llamó Mente Universal, ¿a quién podemos atribuir el objeto de la evolución?

\subsection{El relojero ciego}

Como hemos defendido hasta ahora, el punto fuerte de la teoría evolucionista (teoría que Dennett hará suya) es la afirmación que dice que la evolución actúa (en palabras de Richard Dawkins²) como «un relojero ciego»: la evolución posibilita el caldo de cultivo que permitirá la posibilidad de un auto-desarrollo en los individuos, un desarrollo que no viene delimitado por leyes rígidas ni estrictas que marcan el recorrido, sino que viene delimitado por la imposibilidad de cometer incompatibilidades. Es 


\section{El papel de la evolución en la teoría de Danniel Dennett}

decir, las partículas no pueden mezclarse sin más, sino que suponen una cierta normatividad externa indicada por las enzimas que las componen y que permiten, a la vez que restringen, la posibilidad de unificación en partículas más complejas. Dennett emplea una metáfora: es como si cada partícula tuviera una puerta que sólo puede abrir quien posea la llave. Esta unificación sí que es aleatoria ya que depende del caso concreto de cada partícula el que llegue a unificarse pudiendo constituir así mejores estructuras.

¿Cómo debemos entender este proceso de unificación y desarrollo? Muchos han supuesto que lo que Dennett (y otros evolucionistas como R. Millikan ${ }^{3}$ ) defienden cuando hablan de este tipo de superación biológica es una determinación física. Pero nada más lejos. Dennett apela a la genealogía: del mismo modo que en ingeniería se recurre a la reversión para analizar los proyectos de la competencia, también los organismos recurren a esta estrategia, porque los organismos son productos de diseño creados indirectamente por el entorno en conjunción con su propia distribución interna (producto de su anterior desarrollo), que no responde más que a los intereses de supervivencia que la evolución depositó ciegamente en el inicio. Los organismos se desarrollan según conviene para sobrevivir. Pero esta declaración corre el peligro de ser malinterpretada: lo que Dennett pretende decir es que el organismo no se adapta al ambiente, sino que los organismos se modifican a sí mismos (o son modificados) o se auto-diseñan sólo según el modelo que viene codificado en su genética (que debe ser entendido en un sentido amplio y abierto y no en sentido restringido y cerrado), y si su nueva estructura reacciona bien en el ambiente se verá potenciada por su funcionalidad. Esto es, los organismos parecen comportarse, a menudo, de tal modo que podemos comprender y explicar dicha conducta como intencional (Dennett, 1987a: 27).

Esto desmiente toda pretensión de mitificación del proceso por el cual apareció la mente humana. El ser humano es uno más de los organismos evolucionados. Como todas las demás criaturas, también el hombre comenzó sus andanzas a partir de la ordenación delimitada por los algoritmos de la evolución, sólo que su evolución le ha permitido llegar hasta un punto en el cual parece tener cierto tipo de intereses. Pero dichos intereses no son suyos propios sino que vienen determinados por toda la estructura fenotípica que dicho individuo posee, estructura que (recordemos) en un principio fue pasiva y moldeada por la acumulación aleatoria de los algoritmos de la evolución. Por lo tanto, concluye Dennett, la intencionalidad real o intrínseca que muchos parecen atribuir al ser humano es una ficción y cualquier intento de fundamentarla no es más que 
un intento de creación de ganchos celestes (Dennett, 1995: 102). Si algo explica la existencia de intencionalidad es el proceso evolutivo entendido como progresiva elevación de grados de refinamiento en los fenotipos estructurales de los organismos, elevaciones realizadas mediante grúas concretas (como capacidades añadidas o recursos aleatorios) que permiten una explicación (Dennett, 1995: 116), aunque más desmitificada y desencantada, de cómo realmente llegó el ser humano a poseer una mente capaz de representar objetos. Pero como hemos explicado, dichas representaciones no son directamente elegidas por los individuos sino que son producto de la cognición que posibilita el conjunto evolucionado de su sistema, son producto de la evolución. Por lo tanto, el ser humano sólo puede disponer de una intencionalidad derivada.

\subsection{Criaturas biológicas y creaturas $I A$}

Dennett comienza por denunciar los 25 años durante los que el cognitivismo pretendió ser la única respuesta posible al problema de la mente ${ }^{4}$. Este Computacionalismo de Alta Iglesia (Dennett, 1987b: 60) pretendía responder de modo mecánico a las pretensiones de simulación de capacidades cognitivas humanas, indicando que los estados mentales y la conducta de los sujetos se debía al procesamiento de fórmulas sintácticas capaces de relacionar de modo causal entradas y salidas de datos de modo unidireccional. Pero esta ortodoxia no es capaz de salvar dos lagunas. En primer lugar, no logra salvar el problema que plantea el (llamado) cuello de botella de von Newmann ${ }^{5}$ ("el procesamiento de información simbólica se basa en reglas secuenciales que se aplican una por vez», Varela, 1990: 55). Si los humanos procesaran las órdenes mentales de una en una, el procesamiento de información se ralentizaría, algo que desmiente la intuición que indica que un humano procesa la información muy rápidamente. En segundo lugar, el cognitivismo también considera que «el procesamiento simbólico está localizado» (Valera, 1990: 55), que cierta información se encuentra en sitios localizables del cerebro, algo falso (como veremos) porque cuando se dañan ciertas partes del cerebro, el procesamiento de información encuentra vías alternativas de comunicación ${ }^{6}$. Estas lagunas pueden solucionarse si en lugar de establecer sistemas lineales de procesamiento de información se opta por un sistema de procesamiento distribuido en paralelo, un sistema que permite el procesamiento de distintos algoritmos al mismo tiempo. Aunque la solución de Dennett parte de este supuesto, su solución pasará por considerar un 


\section{El papel de la evolución en la teoría de Danniel Dennett}

centro de conciencia no localizado ni centralizado sino distribuido (e implementado) por toda la red cerebral y neuronal ${ }^{7}$.

Como hemos dicho, Dennett insiste en la afirmación que defiende al humano como un ser intencional ${ }^{8}$. Los humanos son sistemas más o menos complejos de células cuyo comportamiento puede ser analizado, comprendido y explicado como respondiendo a cierto tipo de creencias y deseos (y otros estados mentales). El problema surge cuando intentamos unir esta tesis de la existencia de sistemas intencionales con la polémica tesis dennettiana que afirma que los humanos son también artefactos, sólo que desarrollados según los algoritmos de la ley de la evolución. Entonces, como cualquier artefacto, el ser humano tan sólo posee una intencionalidad derivada. Esto es, esos deseos, razones, creencias, etc., a las que parece responder deben ser extrínsecas al sujeto.

El problema principal que plantea esta teoría derivada de la intencionalidad es establecer el punto en el que es representada: podemos solucionarlo diciendo que los propósitos de la Naturaleza son inexpresados (como hace Millikan), que nosotros somos la cadena y no un eslabón más.

Pero entonces, ¿qué decir de los objetos que tienen una función determinada? Que es sólo ilusoria. Afirmar que algo tiene la función para la cual fue construido (y que no puede cumplir ningún otro propósito) es equivocarnos, caemos en la falacia intencional. La realidad es que la significación no es intrínseca ni natural, sino funcional: la función de algo viene dado por su propia historia, ya que puede ser interpretada de varios modos según su contexto causal. Del mismo modo, lo que significa depende de la época. Pero, ¿no corremos peligro de caer en el adaptacionismo lamarckiano al afirmar que algo se adapta a la función según conviene? No, porque no estamos hablando de adaptación. Algo puede no haber sido diseñado para una función concreta, pero si ahora realiza bien una tarea que no le corresponde, entonces puede ser empleado para dicha función (por ejemplo, un dispositivo detector de billetes de metro, el pulgar del panda, el pulmón). Es decir, que lo que hace la evolución no es tanto dotar a sus productos de una adaptabilidad, sino que más bien actúa como un «chapuzas»: lo que la evolución hace es bricolaje. La Naturaleza corta y pega aquello que funciona adecuadamente para alguna función concreta y lo recoloca en el lugar que más provecho puede proporcionar. No hay intencionalidad original, por lo que el realismo de los significados sólo es posible si aceptamos la teoría natural de las funciones como realista.

Dennett, así, afirma que todos los organismos son el resultado de la evolución, marcada por una serie de ciegos procesos de acumulación, de- 
marcados por algoritmos, que les permiten llegar a tener una cierta intencionalidad derivada. Pero, ¿cómo funcionan dichos algoritmos? ¿Cómo se organizan inicialmente dichas unidades mínimas de intencionalidad para poder formar mejores y más complejos sistemas?

\section{Tipos de mentes: criaturas creadas}

Dennett propone un marco en el que colocar las diversas opciones de diseño: es la llamada torre de la generación y la prueba $a^{9}$. Como he indicado anteriormente, la evolución organiza un conjunto de células en diversos modelos según la felicidad (satisfacing) de sus respuestas: una respuesta adecuada (lo que no significa que sea la única, o la mejor, o la más correcta) es aquella que funciona, y una conducta que funciona se verá potenciada y también se verá reforzada la estructura capaz de ocasionarla, beneficiando y maximizando por ello sus posibilidades de desarrollo, mejorando las posibilidades de que dicha respuesta satisfactoria vuelva a repetirse y sea así (re-)compensada. La evolución tiene por objetivo la potenciación ciega de aquellos organismos capaces de realizar los mejores movimientos, los más eficaces. Es decir, la evolución potenciará las estructuras de aquellos organismos capaces de producir futuro, capaces de posibilitar nuevas vías de acción. Por ello, en cada estrato de la torre podemos colocar multitud de clases de organismos (que incluso llegan a solaparse, en el sentido de compartir el 99,9\% de su diseño) capaces de encontrar y realizar movimientos tales que les permitan desenvolverse en su entorno, lo que permitirá la expansión de su población (crecimiento horizontal); pero sólo aquellos organismos cuyos movimientos son más eficaces verán potenciada su estructura de tal modo que serán capaces de sobrevivir y evolucionar (crecimiento vertical). Pero, ¿cómo distinguir estratos?

\subsection{Primer escalón: las criaturas darwinianas}

Este primer estrato de la evolución acoge a aquellos organismos que, ciega y arbitrariamente, fueron generados por selección natural mediante la combinación aleatoria de genes. Aquellos organismos que poseían estructuras mejor diseñadas (que poseían fenotipos estructurales más eficaces) pudieron expandirse y sobrevivir. Dichas estructuras vienen cerradas de antemano y sólo reproducen aquella información codificada genéticamente. 


\section{El papel de la evolución en la teoría de Danniel Dennett}

\subsection{Segundo escalón: las criaturas skinnerianas}

En algunos de esos organismos que fueron capaces de sobrevivir gracias al mejor diseño de sus estructuras, surgió una nueva propiedad: la llamada plasticidad fenotípica. Esto quiere decir que aquellos organismos poseedores de dicha propiedad no estaban genéticamente cerrados, como las criaturas darwinianas, sino que permanecían abiertos. Sus estructuras fenotípicas no estaban diseñadas por completo: ciertas partes de ellas podían ajustarse según lo requirieran los sucesos externos, permitiendo así una mayor eficacia en sus respuestas.

Cuando ya no actúa la herencia genética, es la modificación abierta y aleatoria de las estructuras fenotípicas la que debe responder satisfactoriamente al estímulo externo. Así, se potenciará aquella conducta que mayor positividad externa proporcione. $\mathrm{O}$ mejor aún, los estímulos positivos externos serán los causantes de la potenciación de ciertas conductas y del refuerzo de la estructura que las sustenta. Una vez que alguna de las primigenias conductas lanzadas ciega y arbitrariamente según los dictados de la evolución ha sido ajustada fenotípicamente por el organismo y es reforzada por el entorno mediante la positividad de su respuesta, la conducta que automáticamente realizará el organismo cada vez que sea necesario será aquella que fue en su momento potenciada porque es la que mejor responde a la situación dada.

\subsection{Tercer escalón: las criaturas popperianas}

El ensayo-error es eficaz pero muy arriesgado: puede salir bien siempre que nuestros primeros intentos fenotípicos estructurales sean eficaces, algo poco probable teniendo en cuenta que, probabilísticamente hablando, es mucho más factible encontrar un modo de constitución defectuosa que una efectiva, por lo que es mayor la probabilidad de perecer que de sobrevivir. A ojos vista, será mejor un sistema capaz de preseleccionar aquellos comportamientos eficaces y separarlos de aquellos ineficaces minimizando así el alto riesgo de perecer y extinguirse que supone el método de ensayo-error.

Pero, ¿por qué denominar popperianas a estas criaturas? Porque, como dijo Popper de las teorías científicas, esta mejora de diseño «permite que nuestras hipótesis mueran en lugar de morir nosotros» (Dennett, 1996a: 109). Los organismos popperianos adquieren la capacidad de modificar ellos mismos sus propias estructuras fenotípicas, adquieren la ca- 
pacidad de fijación postnatal del diseño. En condiciones normales, no hay razón para que el organismo no realice dicha acción, dados los suficientes datos (puede haber error, o coacción o prudencia).

\subsection{Cuarto escalón: las criaturas gregorianas}

Llegados a un cierto punto de la cadena evolutiva, aparecen cierto tipo de criaturas capaces de fabricar cierto tipo de herramientas conceptuales que les permiten ir más allá de la mera determinación natural, porque se requiere inteligencia para inventar/fabricar, reconocer y mantener algo como instrumento útil, pero además el uso de dicha herramienta otorga la misma inteligencia a aquellos que la emplean sin haberla creado. Cuanta más información requiera el diseño de una herramienta, mayor inteligencia potencial confiere al usuario. Las herramientas más notables son aquellas denominadas mentales: las palabras.

Las criaturas skinnerianas preguntan «¿Qué voy a hacer a continuación?»; las criaturas popperianas, "¿Qué debo pensar a continuación?». Pero las criaturas gregorianas se aúpan a la humanidad en tanto que son capaces de cooperar. Los organismos gregorianos son capaces de beneficiarse de la experiencia encarnada en las herramientas (especialmente las mentales) que la comunidad social en conjunto ha ido elaborando y transmitiendo a lo largo de los años, lo que les permite reflexionar mejor acerca de lo que deben hacer o pensar a continuación, (les permite aprender «... a pensar mejor sobre lo que deben pensar a propósito del siguiente movimiento» (Dennett, 1995: 624)), consiguiendo así crear una serie de reflexiones internas (meta-representaciones). El organismo gregoriano, así, es capaz de modificar su estructura fenotípica de modo que le permita crear, identificar y generalizar relaciones no existentes en el mundo externo, pero que le serán de gran utilidad por medio del simple uso de conceptos.

La diferencia entre las criaturas (gregorianas) humanas y las criaturas no-humanas consiste en el manejo más eficaz que tienen las primeras de pensamientos concretos referentes a objetos del mundo que se constituyen de forma abstracta como representaciones. Pero dichas representaciones no son individuales, sino que vienen posibilitadas por el entorno social: nadie puede representarse nada más allá de aquello que su entorno le dice mediante sus herramientas mentales. Las criaturas skinnerianas y popperianas pueden actuar tan sólo como si tuvieran pen- 


\section{El papel de la evolución en la teoría de Danniel Dennett}

samientos concretos, como si reconocieran objetos del mundo, como si se escondieran agazapadas tras las ramas de un helecho con la intención de sorprender a su presa. Los dos tipos de intención pueden llevar a cometer errores, pero sólo en el primer caso puede llevar a la representación de una proposición falsa creyendo que es verdadera.

En los animales skinnerianos y popperianos podemos adivinar multitud de lagunas en cuanto a representación y generalización porque no tienen pensamiento más allá de su modo de procesamiento central. En cambio, los organismos gregorianos humanos son capaces de pensamiento aún a pesar de perder parte de sus capacidades localizadas porque su estructura cognitiva no está centralizada sino que se compone de transductores periféricos en los que se delega parte de las competencias con el propósito de permitir al agente economizar tiempo de transacción episté mica.

Los organismos gregorianos son especiales porque son capaces de construir hipótesis y representaciones generales acerca de la identidad de los objetos e individuos externos; somos capaces de rastrear objetos según su relevancia en el entorno cambiante y de percatarnos de nuestras acciones porque todo ello lo asociamos a nuestro esquema intencional. Por tanto, sólo aquella criatura (darwiniana) gregoriana dotada de herramientas mentales que es capaz de representarse los objetos del mundo, es capaz de ser considerada como persona. Pero, ¿cómo es posible que simples organismos evolucionados bajo las leyes de la selección natural hayan llegado a poder construir representaciones de objetos del mundo? ¿Por qué es posible el pensamiento?

\section{Cuando la evolución creó el significado}

En «Conditions of personhood»" ${ }^{10}$, Dennett afirma que para ser considerado persona, un organismo debe ser un sistema intencional de primer orden (aquel sistema de deseos y creencias relacionados con los objetos del mundo que no pueden relacionarse con sus propios deseos y creencias) capaz de dar el salto a un sistema intencional de segundo orden (aquel sistema de deseos y creencias capaz de establecer relaciones, además de con los objetos del mundo, también con sus propios deseos y creencias), o bien dar el salto a un sistema de tercer orden (aquel sistema capaz de querer que otro crea que quiera algo), o bien dar el salto a un sistema intencional de cuarto orden (aquel sistema capaz de querer que otro crea que otro quiere que crea que quiere algo), y así, ad infini- 
tud. El paso importante es el salto de los organismos a los sistemas de segundo orden, porque este segundo nivel permite que los individuos compartan notas, se transmitan información, al poder manipular su propia significación. La comunicación permite a cada individuo «ser beneficiario de las labores cognitivas de los otros, de un modo que nos otorga poderes sin precedentes» (Dennett, 1995: 629). El paso al resto de niveles tan sólo depende de la cantidad de información que sea capaz de manejar un individuo, ya que las operaciones pueden volverse casi automáticas. Pero, ¿cuál es el límite entre aquellas criaturas que son sistemas intencionales de segundo orden y aquellas criaturas que tan sólo muestran intencionalidad aparente?

\subsection{La aparición de la representación}

Como sabemos, ciertos animales son capaces de realizar ciertos tipos de acciones con cierta (aparentemente) intencionalidad, como por ejemplo, el pájaro que se aleja un poco del nido fingiendo que está herido para evitar que el depredador no lo detecte al percibir una presa fácil, o la liebre que con aire provocador se yergue sobre sus patas traseras haciendo saber a su depredador que lo ha localizado y que es inútil intentar atraparla porque los dos saben que sería perder el tiempo porque la liebre es más rápida y la distancia que los separa es demasiado elevada como para poder atraparla, o la gacela thompson que se permite el lujo de dar cabríolicos brincos en un alarde de fanfarronería como haciendo saber al león que es tan rápida que puede perder el tiempo en saltos acrobáticos y que mejor sería desistir y buscar otra presa, pero cuyo fin es disuadir al predador. Estos animales son sistemas intencionales de primer orden pero, ¿pueden ser sistemas de orden superior? Es decir, ¿en qué modo podemos decir que el comportamiento que presentan ciertos animales es el resultado de una estrategia organizada?

Parece que, en principio, las conductas organizadas requieren de un elevado número de estructuras de control semi-independiente coexistiendo en el sistema nervioso, pero la verdad es que no se puede precisar. La respuesta requiere la apelación a una fuerte presión selectiva que obligue al organismo a reorganizar sus estructuras fenotípicas de control de modo que pueda formar otra estructura capaz de llevarlo a la expansión y la supervivencia.

Según Dennett, la respuesta parece ser la oportunidad de comunicación. El lenguaje permite la cooperación entre individuos. Cuando una 


\section{El papel de la evolución en la teoría de Danniel Dennett}

especie entra en disposición del lenguaje, se produce la cooperación, por lo que la honradez total o el egoísmo total no son recomendables: interviene el benegoísmo ${ }^{11}$.

Con la comunicación cooperativa surge la necesidad de representar (mentalmente) su conducta porque surge la necesidad de comunicar explí citamente la intención del comportamiento. Sólo que ciertas criaturas están dotadas de confabulación aproximada, un sustituto que puede pasar por una representación de intención capaz de configurar una lista de opciones que simulen acciones intencionales pero que sólo se activan según el caso aferente-eferente, dando incluso la sensación de que están bajo su control.

Por ejemplo, el primer ñu en oler al león no puede transmitir directamente a los demás dicha información porque carece del modo representacional adecuado para hacerlo, como tampoco puede engañar a los demás miembros de la manada, ni tan siquiera puede hacer uso fraudulento de dicha información: tan sólo puede echar a correr porque su sistema eferente recibe el estímulo aferente "Hay un león ahí», que despierta la respuesta "Correr», y ese es el único modo en que los demás ñus pueden hacer uso de la información del primero (indirectamente a partir de la conducta de uno de los miembros de la manada). Este salir corriendo puede parecer una acción intencional, parece referirse a la intención "Correr. para evitar ser una presa», pero en realidad es una acción derivada porque depende de la determinación de su estructura fenotípica, depende de que su sistema aferente-eferente esté distribuido de éste modo y no depende de su propia voluntad.

La red conceptual que se requiere para que un animal se muestre como si tuviera intencionalidad puede ser completamente diseñada por mecanismos darwinianos o skinnerianos, ya que el aprendizaje $\mathrm{ABC}^{12}$ permite reforzar mediante condicionamientos ciertos comportamientos operantes. Pero tan sólo una criatura capaz de aprender de su experiencia pasada (de su historia cognitiva) puede ser considerada como intencional.

Una criatura popperiana no es más que un sistema intencional de primer orden (capaz de tener deseos y creencias acerca del mundo). Pero tan sólo aquellas criaturas capaces de dar el salto a un sistema de segundo orden (aquellas criaturas capaces de reflexionar sobre sus propios deseos y creencias) serán capaces de forjar verdaderas estrategias intencionales: sólo criaturas gregorianas capaces de manipular representaciones y transmitirlas mediante la constitución de herramientas mentales pueden ser conside radas como verdaderamente intencionales. Dichas criaturas gregorianas disponen del principio comando en equipo: cada individuo es capaz de tener tanto conocimiento del proyecto total como para que todos y cada uno de los 
miembros del equipo puedan improvisar en vistas a la consecución del objetivo en caso de imprevistos. Esta estrategia de orden superior aparece sólo en aquellas criaturas cuya presión evolutiva les obliga a representar proposiciones, lo que les proporciona la posibilidad de forjar herramientas conceptuales con las que intercambiar información y poder cooperar.

Dennett hace suya una famosa teoría ${ }^{13}$. Andy Clark y Annette Karmiloff-Smith ${ }^{14}$ distinguen dos tipos de criaturas: aquellas que poseen un cerebro con el conocimiento encarnado (anteriormente explicado) y aquellas criaturas que poseen un cerebro capaz de enriquecerse internamente porque es capaz de volverse a representar (siempre que fuera necesario) el conocimiento que anteriormente se había representado. $\mathrm{O}$ sea, es una criatura con un cerebro capaz de recuperar memorísticamente aquellas experiencias que ya había tenido. Distinguen entre criaturas que tan sólo responden medio-instintivamente a estímulos y aquellas criaturas que son capaces de emplear estratégicamente aquel conocimiento (representado) que ya había tenido anteriormente (apelando así a su propia historia cognitiva y experiencial del mundo).

Es decir, Clark y Karmiloff-Smith distinguen entre aquellas criaturas que tienen conocimiento en el sistema y aquellas criaturas que tienen conocimiento para el sistema. Las primeras son criaturas que responden al condicionamiento operante que les ofrece el entorno; las segundas son criaturas capaces de manipular sus propias representaciones, reproducirlas, combi narlas e, incluso, innovarlas; son capaces de verlas como herramientas (mentales) y de emplearlas como tales para su propio beneficio, y el de su especie. Las segundas son criaturas capaces de manipulación simbólica: el ser humano es capaz de guiarse por sus estructuras sintácticas al mismo tiempo que es capaz de comprenderlas, ya que son representaciones simbólicas cargadas de significación e información. Pero, ¿cómo es capaz el humano de lle gar a comprender? ¿Cómo puede una criatura sintáctica dar el salto a la semántica? ¿Cómo puede un artefacto evolucionado pasar de movimientos sin sentido a significar su conducta? El hombre adquiere la capacidad de comprender y manejar significados porque es capaz de descargar la mayoría de teorías cognitivas en su propio entorno, proyectando hacia el exterior toda aquella información útil y relevante.

\subsection{Fabricando nuevas herramientas: la irrupción de la semántica}

Como hemos dicho, Dennett defiende la teoría que afirma que la evolución de los diseños permite a cierto tipo de criaturas privilegiadas pro- 
El papel de la evolución en la teoría de Danniel Dennett

yectar en símbolos externos la mayor parte de la información. Es decir, adoptan la capacidad de construir dispositivos periféricos para almacenar, procesar y volver a representar, cada vez que sea necesario, aquellos significados relevantes. El ser humano tiene la capacidad de confeccionar símbolos/herramientas conceptuales que transmiten información y que se colocan en el exterior (constituyendo lenguaje) para su posterior uso. Existe la posibilidad de que cierto tipo de criaturas skinnerianas o popperianas puedan transmitir información mediante este tipo de codificación externa (como por ejemplo el rastro de feromonas que las hormigas emplean para señalar su camino o las feromonas que contiene la orina de ciertos animales para la delimitación de su territorio), pero dicha operación se produce de modo operante y condicionado, no mediante reflexión. comprensiva.

En pocas palabras, Dennett defiende una especie de nominalismo. El ser humano es capaz de etiquetar las cosas, pero no de cualquier modo. Dicho etiquetado se refiere a aquello que es significativo, a aquello capaz de ser encuadrado en sistemas externos de almacenamiento de información. No es necesario que sea lenguaje. Ciertas especies se sirven de marcas externas como, por ejemplo, la posición del sol para orientarse y los restos desenterrados de las semillas ya consumidas (como el cascanueces de Clark $)^{15}$. La herencia cultural proporciona autopistas culturales que permiten extender nuestra mente al mundo.

Las criaturas popperianas y gregorianas tienen dos dimensiones de entorno: la interna y la externa. El entorno interno sobrevive precisamente porque es regular: aquellas estructuras fenotípicas funcionales tienden a perpetuarse y expandirse. En cambio, el entorno externo está en continua variación, por lo que cambia aquello relevante y el organismo debe rediseñarse para poder captarlo. Una criatura necesita para sobrevivir poder identificar y discriminar aquello que es relevante de lo que no lo es. Ello sólo es posible mediante el etiquetado de aquello que es relevante. Los símbolos externos sólo tienen significado porque se refuerzan con recursos internos que permiten su identificación: el significado sólo tiene importancia porque hay alguien capaz de captarlo. De este modo, las representaciones pueden llegar a convertirse en objetos mentales.

Por ejemplo, en una biblioteca se emplea un código que permite seleccionar y clasificar en secciones temáticas cada uno de los ejemplares de los que se compone, pero ¿qué sentido puede tener esto? También podríamos diseñar un artefacto capaz de clasificar los libros de una biblioteca según las palabras de su título, ¿no? No, porque necesitamos de 
una criatura capaz de comprender los aspectos importantes que permiten una clasificación lo más correcta o refinada posible porque esos símbolos permiten la rapidez y correcto ajuste del procesamiento de información. Es decir, si la conducta de ciertas criaturas de exportar al entorno externo información ha sobrevivido es porque ello es funcional, ya que permite agilizar el reconocimiento de aspectos y mejorar la estructura cognitiva. Todo ello conlleva el refinamiento de cierto tipo de tecnologías de re-representación: aquí hablamos de meta-representaciones. Se potencia el modo más adecuado y eficaz de clasificar, manipular y reflexionar acerca de las representaciones previas. La estrategia más potenciada es aquella que permite construir herramientas externas cargadas de información y significación pero que a la vez requiere mejorar las propias estructuras fenotípicas con el objeto de reconocer y percibir correctamente dichas re presentaciones, a pesar del constante cambio exterior.

Esto nos viene a decir que lo necesario para la supervivencia de uno de estos organismos no es conocer todas las características del exterior, sino que en el momento adecuado sea capaz de hacer uso de la información exterior porque ha conseguido modificar sus estructuras cognitivas de modo adecuado, ha sido capaz de prever adecuadamente el próximo movimiento. Esto permite que aquellas criaturas capaces de rediseñarse adecuadamente sean capaces de construir redes conceptuales capaces de relacionar su mundo interno con el externo.

Con la aparición del lenguaje, las criaturas gregorianas se dotan de palabras, y las palabras identifican (como etiquetas) aquella información depositada en el entorno externo. En lugar de lenguaje escrito del pensamiento deberíamos hablar de lenguaje hablado del pensamiento ${ }^{16}$.

El niño aprende expresiones a medio entender. Escucha sonidos, los recuerda y repite, incluso los comenta. La repetición de palabras las llega a hacer familiares y la familiaridad permite identificar el sentido de una etiqueta. Así se establecen asociaciones y se potencian las ya establecidas.

La contemplación de representaciones permite recordar la significación que importa, entendemos la información constituyendo conceptos. Las palabras son conceptos primigenios, vocalizados y encarnados que podemos manipular para convertirlos en objetos mentales sobre los que deliberar. Pero, ¿cómo saber qué concepto emplear para según que situación? El hombre construye tecnologías: toda una red de habilidades estructuradas que permite captar y clasificar su entorno según su propia escala de valores, según sea significativo. Es aquí donde podemos ver el importante papel de la cooperación que permite el lenguaje: una vez los 
organismos han llegado al punto de representarse los objetos del mundo, establecer relaciones entre ellos y comunicarlo a los demás (lo que indica que son relevantes se procede al establecimiento de hipótesis que permitan prever el futuro. Se constituye la ciencia: «una vez que tenemos lenguaje - una caja repleta de herramientas mentales- podemos utilizar estas herramientas en la estructura que genera y que somete a prueba, de manera premeditada y con visión de futuro, conocida como ciencia» (Dennet, 1995: 626). La ciencia permite establecer leyes mediante la generalización y establecimiento de relaciones causales que (a pesar de ser ficticias) permiten al hombre tener la ilusión de poder predecir acontecimientos. La ciencia no está pensada para minimizar los errores, sino para que en caso de cometerlos, los errores se cometan en público, permitiendo la puesta en común de dificultades y escollos con el fin de que los demás miembros de la comunidad puedan llegar a una mayor precisión y refinamiento de las hipótesis.

La principal crítica a esta afirmación es la tesis que afirma que ciertos seres están sometidos a un cierre cognitivo. Esta tesis, defendida por Chomsky, Fodor o McGinn afirma que, como somos subjetivistas y tenemos un acceso privilegiado de primera persona a ciertas cosas, hay conocimientos que están vedados a ciertos individuos porque no poseen los instrumentos cognitivos adecuados para captar ciertos aspectos, por lo que parte del mundo les será desconocido. Dennett niega esta posibilidad del sentido común afirmando que no existe cierre cognitivo y que un ser humano (como cualquier otro organismo evolucionado correctamente) podría llegar a conocerlo todo, pero que la entropía no se lo permite porque destruirá siempre al organismo antes de que éste llegue a poseer el conocimiento total. Parece que esta tesis dennettiana defiende la posibilidad de una clausura física del mundo ${ }^{17}$, algo que también se contrapone al sentido común.

\section{Bibliografía}

DENNETT, DANIEL: Brainstorms: philosophical essays on mind and psychology, Bradford Books, 1978a (se emplea la reedición de Harvester Press de 1986).

- "Current issues in the philosophy of mind», American Philosophical Quarterly, vol. $15, \mathrm{n}^{\circ} 4,1978 \mathrm{~b}$.

- Elbow room: the varieties of free will worth wanting, MIT Press, 1984a (trad. cast. La libertad de acción, Gedisa, 1992).

- «I could not have done otherwise -so what?», The Journal of Philosophy, 81, 1984b, pp.553-567 (trad. cast. «No podría haber actuado de otro modo, ¿y qué?», en Corbí y Moya (eds.) (1999), pp. 133-143). 
- "Cognitive wheels: the frame problem of AI», en Ch. Hookway (ed.), Minds, machines and evolution, Cambridge University Press, 1984c.

- The intentional stance, MIT Press, 1987a (trad. cast. en Gedisa, 1991).

- "The logical geography of computational approaches: a view from the East Pole», en M. Brand and M. Harnish (eds.), Problems in the representation of knowledge, University of Arizona press, 1987b, pp. 59-79.

- "The interpretation of texts, people and other artifacts", Philosophy and Phenomenological Research, 50, supplement, 1990, pp. 177-194.

- Conciousness explained, Little Brown, 1991 (trad. cast. en Paidós, 1995).

- «Temporal anomalies of conciousness», en Y Christen and P. S. Churchland (eds.), Neurophilosophy and Alzheimer's disease, Springer-Verlag, 1992.

- "The role of langague in intelligence», en J. Khalfa (ed.), What is intelligence?, Cambridsge University Press, 1994b.

- Darwin's dangerous idea: evolution and the meaning of life, Simon and Schuster, 1995 (trad. cast. en Galaxia Gutenberg, 2000).

- Kinds of minds: toward an understanding of consciuoness, Basic Books, 1996a (trad. cast. en Debate, 2000).

- «How to do other things with words» Royal Institute Conference on Philosophy and Languague, en J. Preston (ed.) Philosophy, 42, supplement, 1997, pp. 219-235.

- Freedom evolves, Viking Penguin, 2003 (trad. cast. en Paidós, 2004).

- and J. HaUgeland, "Intencionality», en R. L. Gregory (ed.), The Oxford Companion to the mind, Oxford University Press, 1987.

Varela, Francisco: Conocer, Gedisa, Barcelona, 1990.

\section{Notas}

1 Dennett (1996a: 102).

2 R. Dawkins: The blind watchmaker, Longmans, 1986 (trad. cast. en Labor, 1989).

${ }^{3}$ El lector interesado en las teorías de R. Millikan puede consultar Language, thought and other biological categories, MIT Press, 1984 y White Queen psychology and other essays for Alice, MIT Press, 1993.

4 El lector interesado en el tratamiento que Dennett realiza de otras aproximaciones a dicho problema puede consultar Dennett (1978b).

5 Este problema lo plantea por primera vez John von Newmann con la intención de reformular la llamada máquina de Turing. Una máquina de Turing es una estrutura sintáctica conceptualmente simple capaz de realizar funciones de computación mediante la entrada y salida de datos. Pero dicha máquina sólo realiza un movimiento por vez, por lo que el proceso es lento para cómputos complicados. Cf. A. Turing, «On computable numbrers, with and application to the Entscheidungsproblem", Proceedings of the London Mathematical Society, vol. XLII (1937), pp. 230-265 y J. von Newmann, The computer and the brain, Yale University Press, 1958.

${ }^{6}$ Para la revisión que hace Dennett de ciertas anomalías cerebrales puede verse Dennett (1992).

7 Dennett (1991), especialmente el capítulo 9.

8 El texto básico sobre este tema es «Intentional systems», en Dennett (1987a).

9 Dennett (1996a: 101ss.) 


\section{El papel de la evolución en la teoría de Danniel Dennett}

10 Este y otros artículos pueden encontrarse en Dennett (1978a).

11 El término benegoísmo (benegoism) es acuñado por Dennett para referirse al comportamiento que los individuos adquieren cuando se encuentran en una situación límite. Cuando no queda más remedio que cooperar o perecer, los organismos no tienen otra salida que forjar estrategias conjuntas que les permitan el más alto grado de supervivencia posible. El término se refiere a unas palabras que (parece ser) pronunció Benjamin Franklin en la reunión previa a la firma de la Declaración de Independencia de los Estados Unidos. El lector interesado en un examen más detallado de este concepto puede consultar Dennett (2003: 221ss.).

12 El aprendizaje ABC (Asociationism, Behavorism, Conexionism) es aquel que permite a un organismo establecer conexiones entre redes comportamentales sencillas mediante la «historia de los refuerzos» que han contribuido a la potenciación de sus conductas satisfactorias.

13 Una primera defensa de esta teoría puede verse en Dennett, «Labeling and learning”, Mind and Language, VIII, 1994, pp. 549-548. Para una versión revisada puede consultarse Dennett (1997).

14 «The cognizers innards: a psychological and philosophical perspectives on development of thought», Mind and Language, 8 (1993), pp. 487-519.

15 Andy Clark, Associative engines: conectionism, concepts and representational change, MIT Press, 1993, citado en Dennett (1996a: 102).

16 La tesis de la existencia de un lenguaje del pensamiento (llamado mentalés) en el que se codifica toda orden cerebral es el punto fuerte de la teoría de la mente del filósofo estadounidense J. A. Fodor. El lector interesado puede ver Psychosemantics, MIT Press, 1987 (trad. cast. en Tecnos, 1994). Puede verse también The language of thought, Crowell, 1975 (trad. cast., Alianza, 1984).

${ }^{17} \mathrm{La}$ tesis de la clausura física del mundo es actualmente defendida por varios autores, entre ellos Jaekwon Kim o David Lewis. Todo hecho del mundo (incluidos los estados mentales de los seres humanos), defienden, tiene una explicación en términos físicos, explicación que (si bien todavía no podemos aportar) la evolución y desarrollo de mejores y más completas teorías científicas permitirá en un futuro. Para una revisión pormenorizada de las tesis de la clausura física del mundo (y una contrarréplica) puede consultarse con provecho el excelente trabajo de Josep E. Corbí y José L. Prades, Minds, causes and mechanisms, Blackwell, 2000. 